Mol. Cryst. Liq. Cryst., 1987, Vol. 149, pp. 303-316

Photocopying permitted by license only

(C) 1987 Gordon and Breach Science Publishers S.A.

Printed in the United States of America

\title{
New Non-Reorientational Optical Nonlinear Effect in MBBA
}

\author{
PEDRO MENÉNDEZ-VALDÉS AND JOSÉ A. MARTIN-PEREDA
}

Universidad Politécnica de Madrid

Escuela Técnica Superior de Ingenieros de Telecomunicación

28040 Madrid - Spain

(Received August 8, 1986; in final form February 27, 1987)

A new effect producing self-focusing of light in a nematic MBBA film is reported. This effect produces a static diffraction pattern composed of circular rings which is different from the ones arising from self-focusing previously reported. The influence of the cell thickness, the optical intensity, and the wavelength is studied. Once the nematic is distorted by a laser beam, the effect produced in other light beam passing through the modified region is independent of its polarization. This isotropic behavior shows that a molecular reorientation has not been produced. The origin of this effect seems to be the same of that of the effect which produces a randomly oscillating diffraction pattern previously reported by our group. Some possible causes such as thermal indexing, convective instabilities and self-induced transparency are discussed.

Keywords: nonlinear optics, nematic, self-focusing, diffraction, state diagram.

\section{INTRODUCTION}

Self-focusing and self-trapping of light have been among the most intensively studied topics in the field of Nonlinear Optics. ${ }^{1,2}$ In particular self-focusing in nematic liquid crystals has been early obtained both in isotropic ${ }^{3}$ and nematic ${ }^{4}$ phases. Its origin has always been a molecular reorientation induced by the optical field. The particular feature of self-focusing in nematics is a very peculiar ring-shaped

$\uparrow$ Paper presented at the 11th International Liquid Crystal Conference, Berkeley, CA, 30 June-4 July, 1986. 
diffraction pattern ${ }^{1,5,6}$ originated from sharp transversal inhomogeneities. This effect has been employed in transverse optical bistability ${ }^{7}$ and opto-optical modulation. ${ }^{8}$ In this last case, the modulated beam must be extraordinary (i.e., polarized as the modulator) because the distortion of the optical axis produce changes only in the phase index of the extraordinary wave. However, experiments with MBBA, as a nematic material, have shown that a weak modulation can also be produced on an ordinary beam. This effect was attributed to a thermal origin and also to a misalignment between both beams which could yield an extraordinary component on the beam.

A new interesting question was outlined when a quasi-chaotic diffraction pattern was observed in MBBA. Over a certain intensity threshold, a new diffraction pattern appeared superimposed on the one due to reorientation. This new diffraction pattern had a much wider angular aperture than the previous one, showing that the modified portion of the liquid crystal was smaller or had a sharper transversal variation. Moreover, the diffraction rings oscillate chaotically, modifying the relative intensity among them. This effect was first observed in homeotropic and hybrid films of MBBA, ${ }^{10}$ and also reported for cylindrical homeotropic structures. ${ }^{11}$ The origin of this effect has been discussed in Ref. 11, and a self-induced transparency of MBBA was suggested. The unstable motion was attributed to the variation of the position of the beam waist-and hence of the bleached region-along the axis, due to the induced refractive index variation. There were, however, several aspects which were not clear, and had to be more carefully studied. In particular, another low intensity laser beam passing through the affected region of the sample was diffracted by it, independently of its polarization, indicative of a non reorientational origin. This fact suggested to isolate it from the reorientation effect in order to study it separately. This can be done by reducing sample thickness.

Such a study is reported in this paper. By reducing molecular reorientational effects in the samples, a static, large aperture diffraction pattern can be observed. Different regions for static, unstable and reorientation effects are delimited depending on laser intensity, cell thickness and wavelength. The origin of this effect is discussed.

\section{EXPERIMENTAL CONDITIONS}

The experiments were carried out as shown in Figure 1. The high power (5 watt, $\mathrm{cw}$ ) polarized $\mathrm{Ar}+$ laser generates the optical field, 


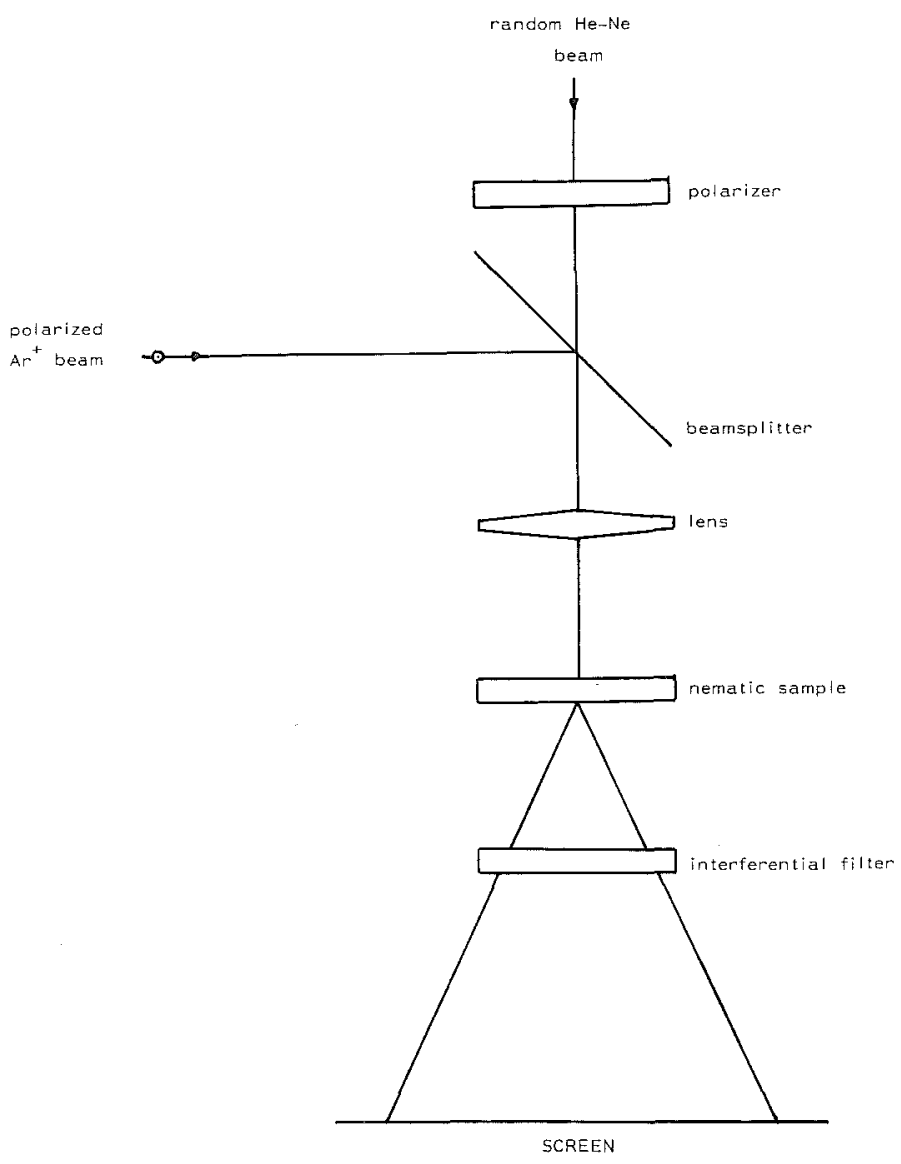

FIGURE 1 Experimental set-up employed.

whereas an $\mathrm{He}-\mathrm{Ne}$ unpolarized laser beam was used as a probe in some experiments. To observe the self-induced diffraction pattern, the $\mathrm{He}-\mathrm{Ne}$ laser is switched off, and the interferential filter removed. A polarizer may optionally be located at the output to analyze the polarization of the diffracted beam.

Both laser beams are focused at the nematic by the same lens. Their waists are not coincident because of the lens chromatic dispersion; however, the nematic region distorted by the argon-ion laser beam is transversed by both beams, coaxially superimposed. Hence, it is inspected by the He-Ne probe beam, which is isolated by the filter afterwards. The polarizer in the $\mathrm{He}-\mathrm{Ne}$ beam path selects either 
parallel or perpendicular polarization with respect to the $\mathrm{Ar}+$ beam, thus testing the induced birrefringence.

Two nematics have been used, namely MBBA and 5CB. Our MBBA had traces of some impurities and was slightly darker, and hence more absorbing, than pure MBBA. As will be shown later, 5CB was employed in order to clarify the difference between the single reorientation observed in it and some other effects obtained in MBBA.

Several cell thicknesses have been used for MBBA. Most of the previously reported works use cell thicknesses over $100 \mu \mathrm{m}$, since, in this way, molecular reorientation is easier to achieve at relatively low powers. In our case, we are trying to minimize this molecular reorientation. Hence, thinner cells, ranging from 10 to $75 \mu \mathrm{m}$ were employed. Homeotropic alignment, induced by chemical pretreatment with $\mathrm{HTAB}$, was used in all cases.

The spatial distribution of light intensity was monitored with a Charge Coupled Device (CCD) and registered on an oscilloscope screen. An optical attenuator (optical density 3) was used in order to protect the CCD from the high laser power.

\section{EXPERIMENTAL RESULTS}

In order to compare the new observed effects, the diffraction pattern produced by the self-focusing of a laser beam in a $100 \mu \mathrm{m}$ thick $5 \mathrm{CB}$ homeotropic cell, where just the reorientation effect is present, has been obtained. Figure 2(a) shows the diffraction pattern due to a weak reorientation in the cell and Fig. 2(b) its spatial intensity distribution as obtained from the CCD. The narrow peaks that appear are due to interference fringes produced by the optical attenuator.

Figure 3(a) shows a completely different diffraction pattern observed in a $36 \mu \mathrm{m}$ film of MBBA. It is composed of two different sets of perfectly circular static rings. When the laser power increases, the thinner rings broaden. The angular aperture of the diffracted beam is considerably wider than in the case of molecular reorientation. Moreover, the effect is obtained at rather lower intensities. Indeed, Fig. 3(a) was obtained at $600 \mathrm{w} / \mathrm{cm}^{2}$ in a $36 \mu \mathrm{m} \mathrm{MBBA}$ sample, while Fig. 3(b) is a reorientational pattern obtained at 15 $\mathrm{kw} / \mathrm{cm}^{2}$ in a $100 \mu \mathrm{m}$ 5CB sample.

Figure 3(c) is the spatial intensity distribution of Figure 3(a). Equally spaced thinner rings are clearly shown in it; thicker rings of this figure cause unequal heights of the peaks. Figure 3(d) shows a typical (seven rings) diffraction pattern produced by reorientation. 


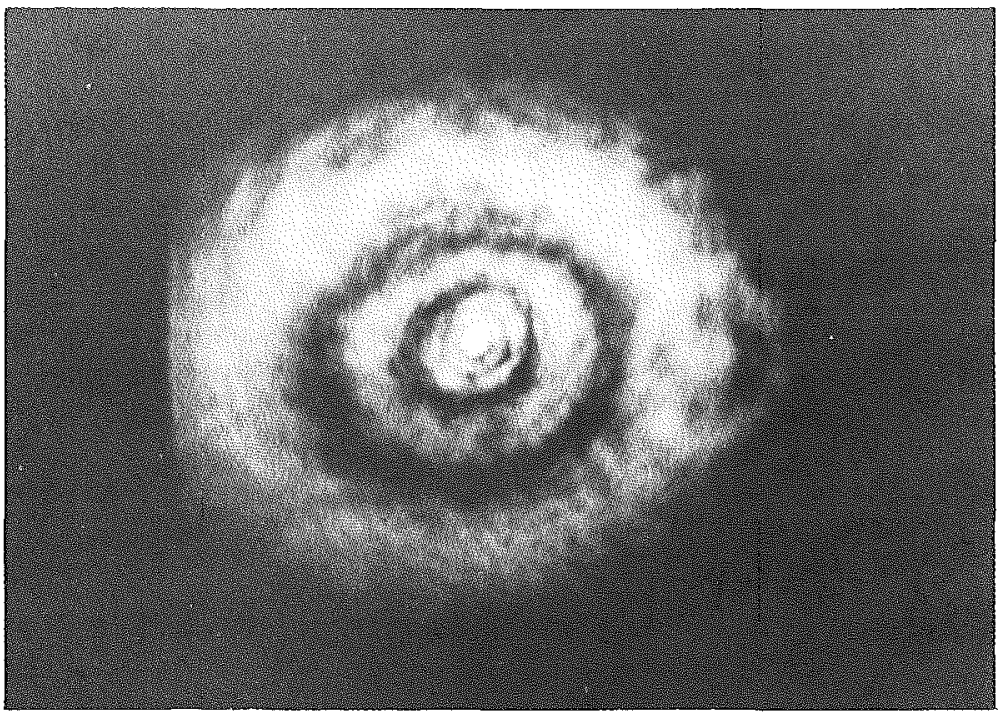

(a)

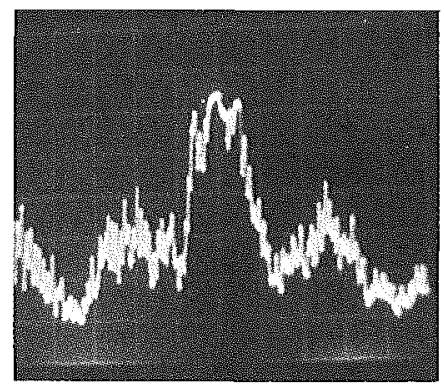

(b)

FIGURE 2 Diffraction pattern due to optically induced reorientation in a $5 \mathrm{CB}, 100$ $\mu \mathrm{m}$, cell. (a) Aspect of a two ring reorientational diffraction pattern. See Color Plate I B. (b) Intensity distribution.

The threshold intensity at which this self-focusing arises shows an inverse dependence on the cell thickness. In the above mentioned case $(36 \mu \mathrm{m})$ its value is $240-300 \mathrm{~W} / \mathrm{cm}^{2}$ for $\lambda$ ranging between 414.5 and $476.5 \mathrm{~nm}$. However, a $12 \mu \mathrm{m}$ sample requires about $1.5 \mathrm{kw} / \mathrm{cm}^{2}$ and just $110-145 \mathrm{~W} / \mathrm{cm}^{2}$. are needed for a $50 \mu \mathrm{m}$ cell at the same wavelengths. Going to higher values of the thickness, this effect is not present, at least as clearly as for smaller values. Reorientation is observed instead. 


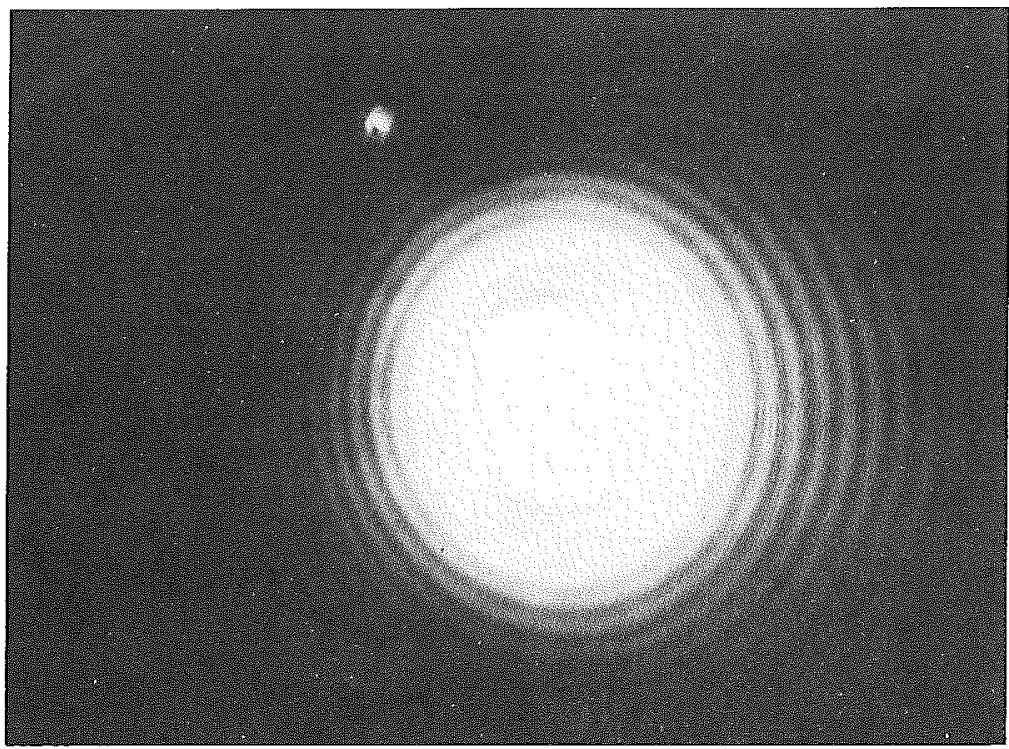

(a)

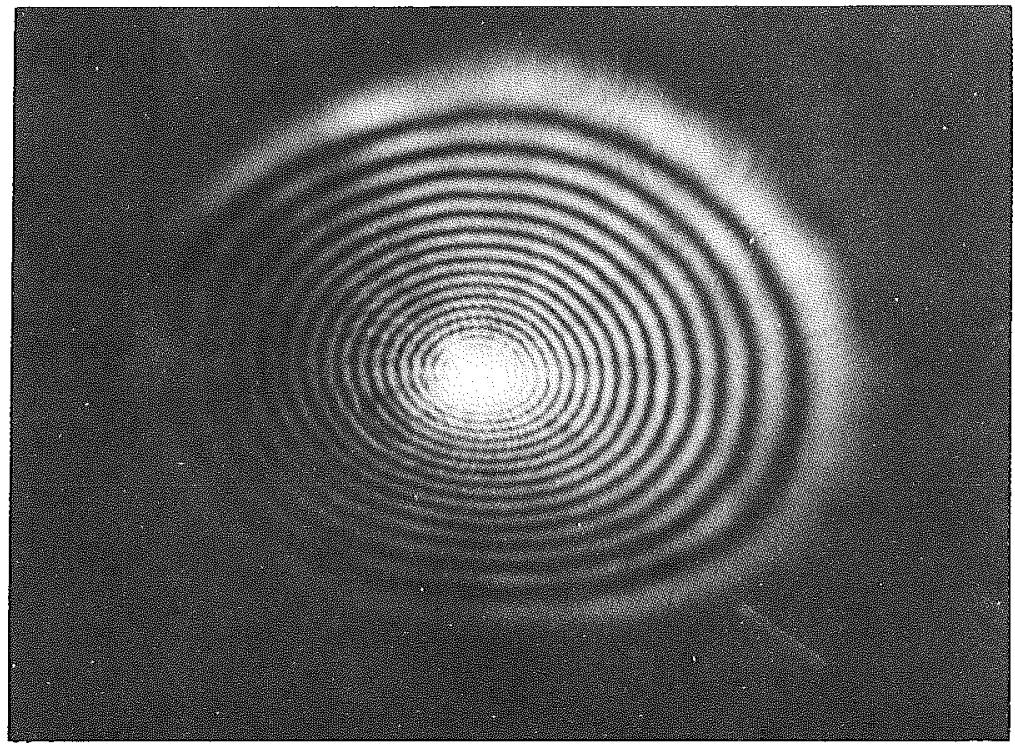

(b)

FIGURE 3 Comparison between (a) the new static diffraction pattern and (b) diffraction pattern due to reorientation. (c) and (d) show spatial intensity distribution of (a) and (b). See Color Plate II. 
NEW OPTICAL NONLINEAR EFFECT IN MBBA

309

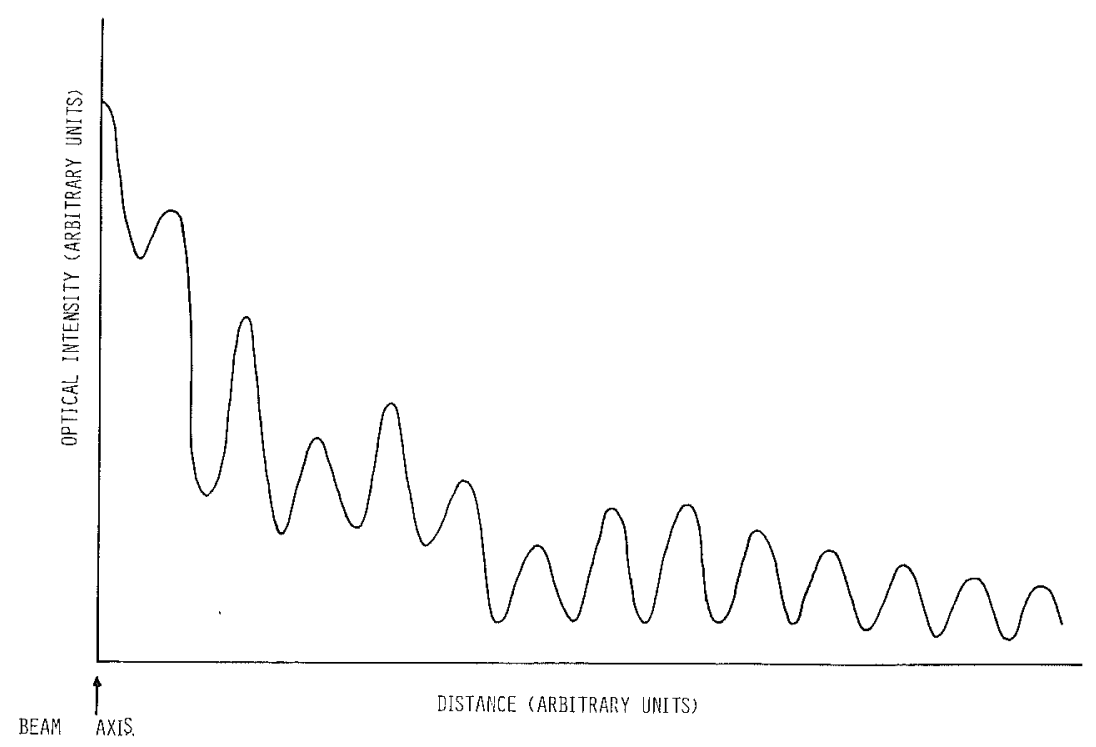

(c)

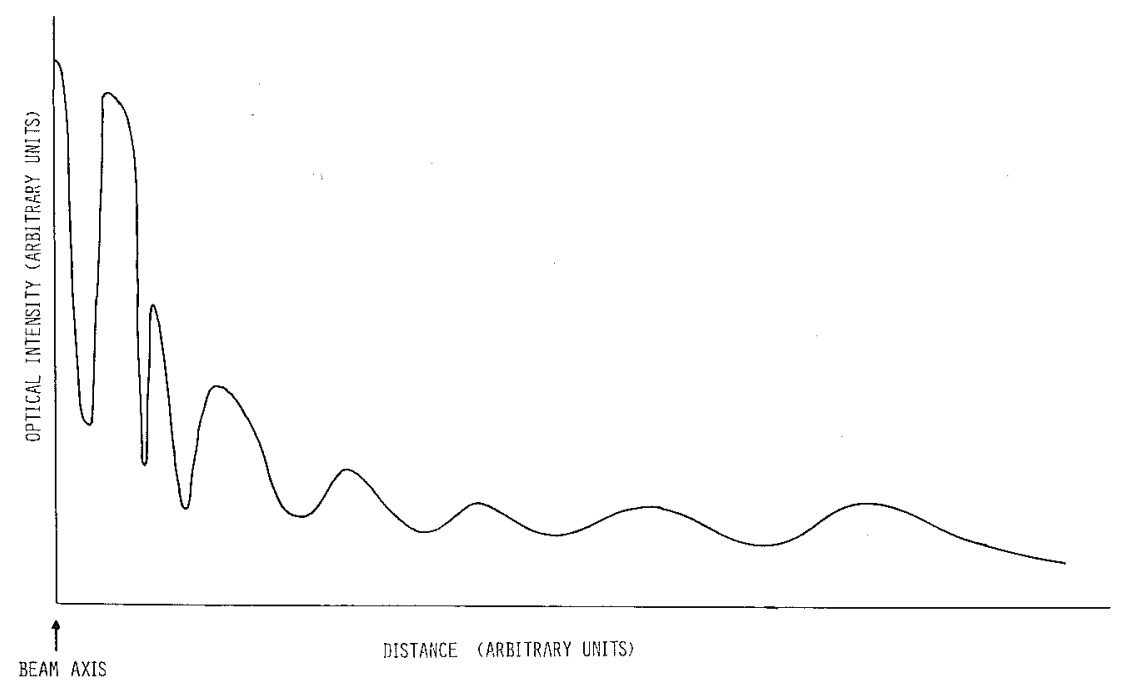

(d)

FIGURE 3 (continued) 
Another fact needs to be pointed out. The diffraction pattern is slightly different for every thickness. In thin samples (10 to $15 \mu \mathrm{m})$ the beam divergence is high, but the annular shape only appears as a slight modulation on an almost homogeneous diffraction pattern. In samples with an intermediate thickness (30 to $40 \mu \mathrm{m})$ the diffracted beam shows, as it can be seen in Figure 3(a), a set of very regular rings. The beam divergence is higher than above. In both cases, if the polarization of the diffracted beam is analyzed, a conoscopic interference pattern of uniaxial media, as shown in Figure 4, is obtained with an output polarizer placed perpendicular to the incident beam polarization.

The previously reported ${ }^{10,11}$ chaotic behavior appears above a certain threshold. A picture of the observed diffraction pattern is shown in Figure 5. Unfortunately, most of the oscillating rings cannot be seen in photographs. However, radial segments are observed in some places, corresponding to prints leaved by some of the moving rings. When this chaotic state is present, the conoscopic cross interference pattern of uniaxial media is again observed if the sample is placed between crossed polarizers. These instabilities are not observed in

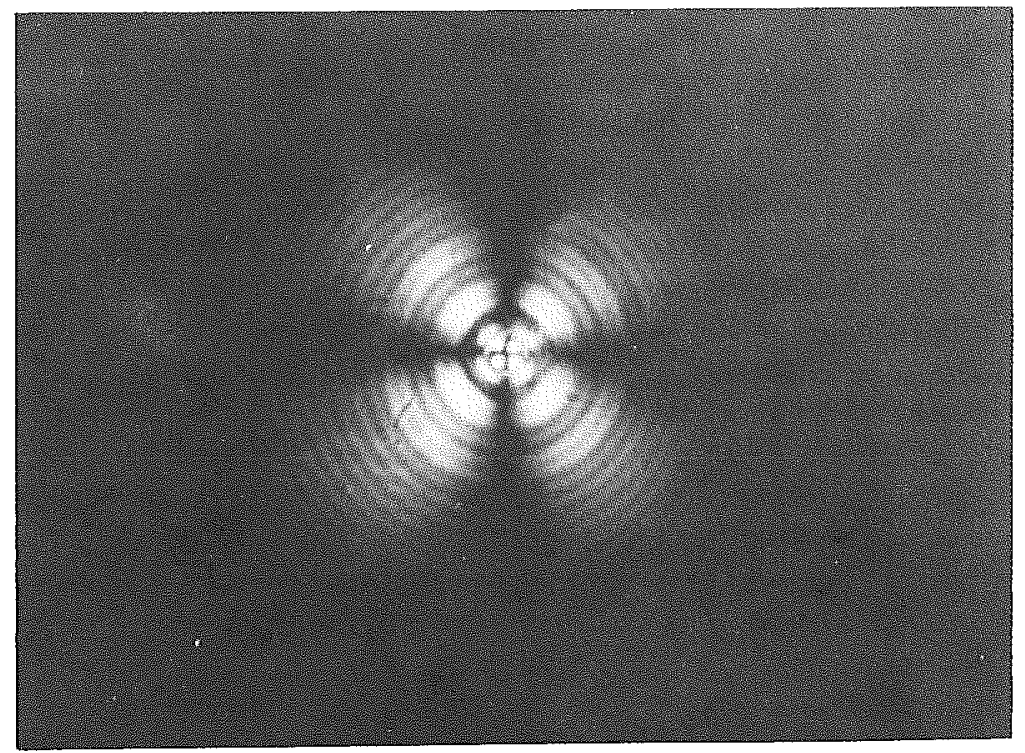

FIGURE 4 Conoscopic interference pattern obtained when the sample is placed between crossed polarizers. See Color Plate III. 


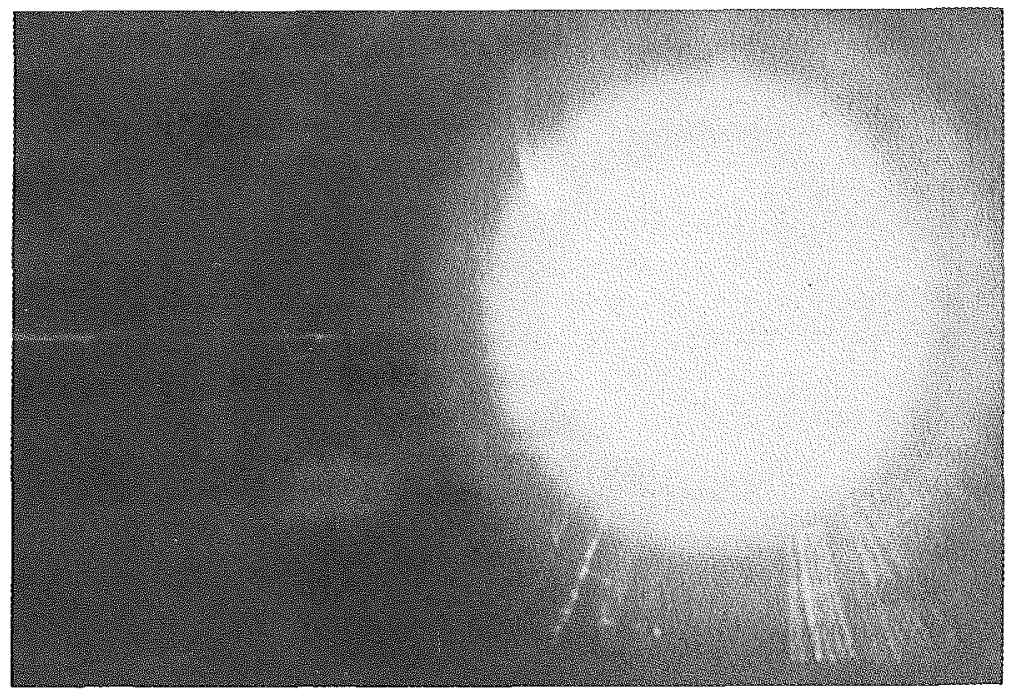

FIGURE 5 Chaotic oscillating diffraction pattern. See Color Plate III.

very thin (less than $20 \mu \mathrm{m}$ ) films. In 20 to $30 \mu \mathrm{m}$ thick films, the effect is metastable: the static diffraction pattern finally appears after 1 or two minutes. In the thickest films used (i.e. 50 to $70 \mu \mathrm{m}$ ), and for intensities below the one causing the unstable effect, the diffraction pattern is typical for reorientation effect. However, only one, or at the most two rings are present before the transition to the chaotic state. The rate of the random oscillations increases as intensity does.

Finally, a new static diffraction pattern (Figure 6) becomes apparent at about $200 \mathrm{w} / \mathrm{cm}^{2}$ for a $50 \mu \mathrm{m}$ thick sample and about 240 $\mathrm{w} / \mathrm{cm}^{2}$ for a $36 \mu \mathrm{m}$ one, at $25^{\circ} \mathrm{C}$. This threshold intensity becomes lower for higher temperatures. The pattern size now decreases for increasing intensities, indicating that the nonlinear effect becomes saturated: increasing the intensities does not produce sharper transversal inhomogeneities, but wider affected regions. In the thinnest films, where no chaotic motion is present, saturation arises directly from the static self-focusing.

The above results have been obtained for $\lambda=514.5 \mathrm{~nm}$ in all the cases where wavelength has not been expressly indicated. Moreover, there is a strong wavelength dependence of these effects. For instance instabilities do not appear for the $632.8 \mathrm{~nm} \mathrm{He}-\mathrm{Ne}$ radiation, using the same or even higher intensity densities. The shorter the wave- 


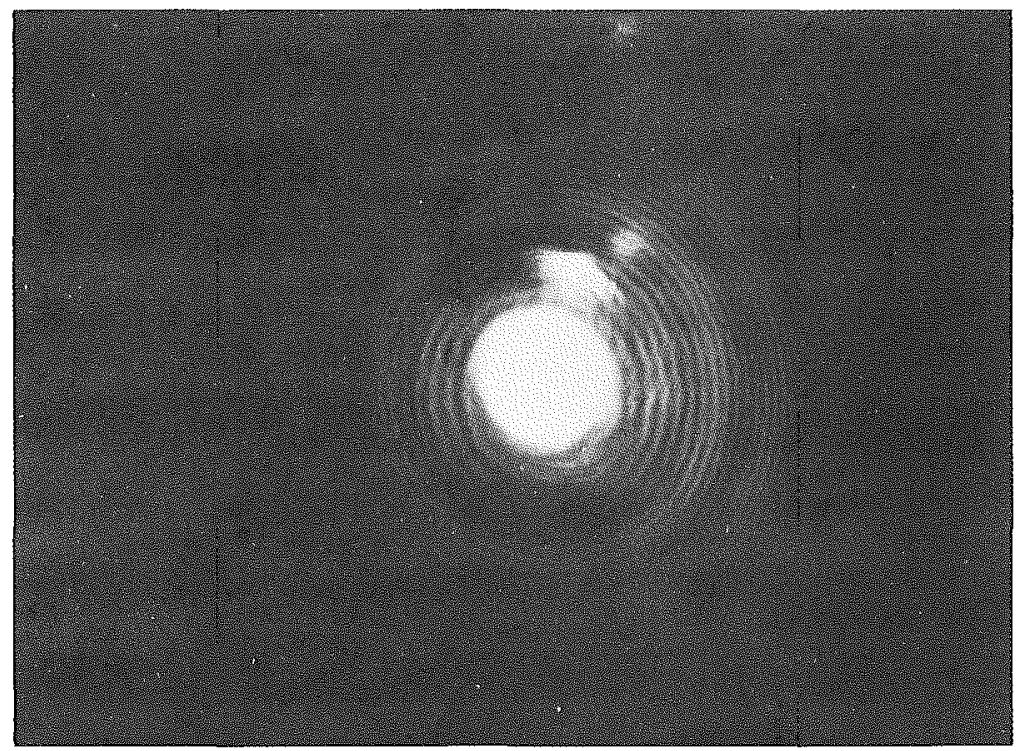

FIGURE 6 Diffraction pattern when saturation begins. See Color Plate IV.

length, or the thicker the film, the lower the intensity at which the transition to the chaotic behavior is produced. A summary of these results is given in Figure 7(a) for a $36 \mu \mathrm{m}$ sample and in Figure 7(b) for $50 \mu \mathrm{m}$. The wavelengths studied correspond to $\mathrm{Ar}^{+}$and $\mathrm{He}-\mathrm{Ne}$ lines, i.e., 457.9, 476.5, 488, 496.5, 414.5 and $632.8 \mathrm{~nm}$. A tentative state diagram is given.

Molecular reorientation can be checked using the probe $\mathrm{He}-\mathrm{Ne}$ laser of Figure 1. If reorientation is present, the probe beam is not diffracted when it is polarized perpendicularly to the $\mathrm{Ar}^{+}$beam polarization. In this case, only the ordinary index is "seen" by the probe, and so inhomogeneities are not detected. This can be clearly observed for samples having a thickness above $50 \mu \mathrm{m}$. The probe is modulated only when its polarization is parallel to the one of the disturbing beam.

Below this thickness, or under chaotic behavior, there is not an appreciable difference between diffraction of parallel and orthogonal polarized probe beams. The chaotic motion, however, cannot be observed in the probe beam. Moreover, the probe beam between crossed polarizers does not show the conoscopic cross interference in the region of static diffraction, but it does it in the region of random motion. Both effects can be attributed to the location of the probe 
beam waist outside the distorted region due to chromatic aberration of the lens.

\section{DISCUSSION OF RESULTS}

The observed conoscopic cross interference as well as the experiments with a probe beam, show that reorientation has not been produced when our diffraction pattern is present. Similar results have been obtained when the diffraction pattern becomes unstable. This suggests the same origin for both effects. In this way, in samples below 50 $\mu \mathrm{m}$, the new effect arises at a certain intensity depending on the cell thickness and the wavelength used. Increasing the intensity causes the effect to become unstable and, finally, to saturate at even higher intensities (numerical data have been given above). When thicknesses over $50 \mu \mathrm{m}$ are employed, reorientation effects can be observed. The new effect arises then only in its unstable form. This is because reorientation is dominant up to the threshold intensity at which instabilities appear. Saturation arises at higher intensities.

The large apertures of the observed diffraction patterns indicate that the origin cannot be a thermal indexing. The heat conduction and convection would induce, in the steady state, smooth transversal inhomogeneities which would yield a certain broadening of the beam, but never such diffraction pattern.

Convective instabilities with cylindrical symmetry have been suggested as another possible origin. As is known, convection equilibrium is reached after a certain time, domains being its final result. However, the response time, as measured by us, is about $2.2 \mathrm{~ms}$ for a $36 \mu \mathrm{m}$ thick cell. This time is much shorter than conventional convective times. Moreover, an anular shaped convective domain with cylindrical symmetry should have radial molecular flow. Under these circumstances, a linearly polarized laser beam should "see" only the ordinary refractive index along a diameter perpendicular to the polarization direction. Any other direction should have an extraordinary component. Therefore, the diffraction pattern would not present circular symmetry. Hence, this solution is not possible.

Any tentative approach to the origin of the effect needs to justify a sharp refractive index variation inside the cell. As this variation does not come from reorientation, one has to suppose that the material has strongly changed its optical properties. This change might be produced by molecules electronic excitation, phase transitions or thermally induced chemical or photochemical alterations. These three possible causes need to be analyzed in a future work. 


\section{$1\left(w / \mathrm{cm}^{2}\right) / 30$}

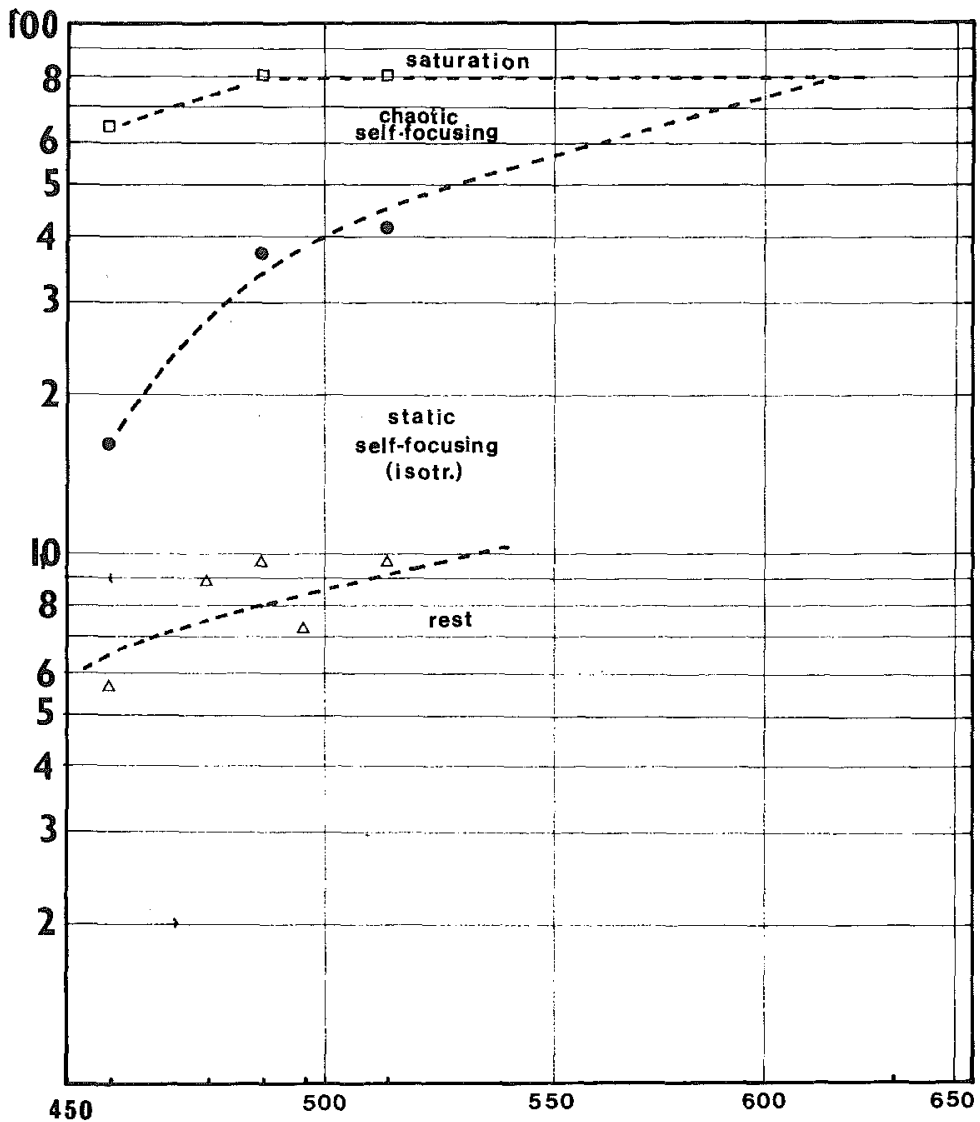

\section{WAVELENGTH $(\mathrm{nm})$}

(a)

FIGURE 7 State diagrams of the observed diffraction pattern. (a) $36 \mu \mathrm{m}$ sample (b) $50 \mu \mathrm{m}$ sample. Points in abscissas are $4579,4765,4880,4965,4145$ and $6328 \AA$. Dashed lines are only qualitative.

\section{CONCLUSIONS}

A new type of self-focusing in homeotropic samples of MBBA has been reported. The result is a new static diffraction pattern composed of circular rings with a different intensity distribution than the one 


\section{$I\left(W / \mathrm{cm}^{2}\right) / 30$}

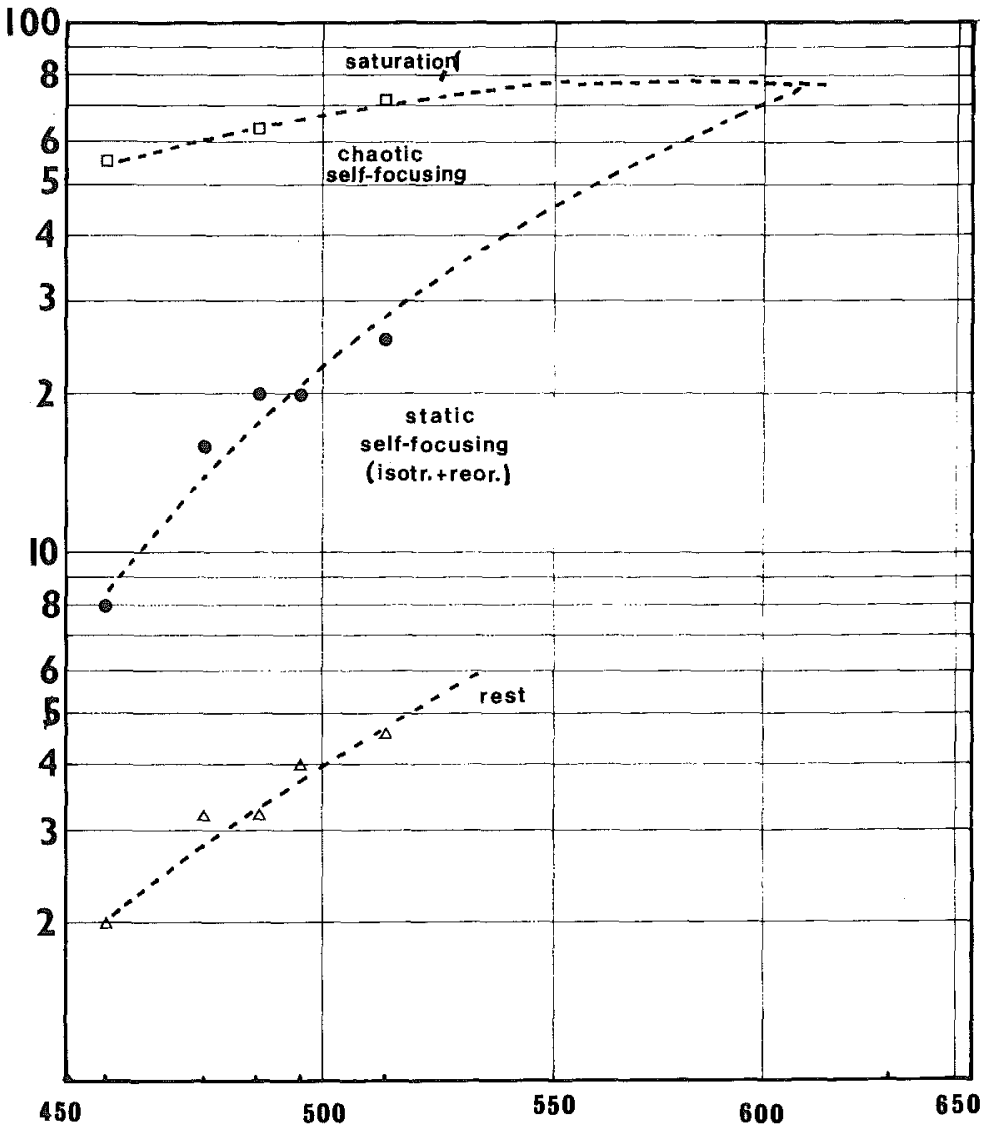

WAVELEHGTH (nm)

(b)

FIGURE 7 (continued)

due to the classical self-focusing from molecular reorientation. The origin of this effect is the same one than that of the previously reported quasi-chaotic diffraction pattern. From several considerations this effect has been shown not to be due to molecular reorientation. Its dependence on cell thickness, laser beam intensity and wavelength has been studied. Some possible origins have been discussed showing 
that interpretations involving thermal indexing and convective instabilities are not in good agreement with the results. A sharp variation in the optical properties of a small region at the beam waist has been suggested to be due to electronic excitation, chemical alteration, or phase transition. New experiments in order to discern the adequate solution are in progress in our group.

\section{Acknowledgments}

The authors thank Dr. Oton's comments, suggestions and help. Financial support has been provided by the Spanish Comisión Asesora de Investigación Cientifica y Técnica, Grant No. 1564/82.

\section{References}

1. Y. R. Shen, The Principles of Nonlinear Optics, John Wiley and Sons, New York (1984).

2. F. A. Hopf and G. I. Stegeman, Applied Classical Electrodynamics. Vol. 2: Nonlinear Optics, John Wiley and Sons, New York (1986).

3. D. V. G. L. Narashima Rao and S. Jayaraman, Appl. Phys. Lett., 23, 539 (1973).

4. B. Ya Zel'dovich and N. V. Tabiryan, Sov. Phys. JETP, 52, $1210(1980)$; N. F. Pilipetski, A. V. Sukhov, N. V. Tabiryan and B. Ya Zel'dovich, Opt. Commun., 37, 280 (1981).

5. S. D. Durbin, S. M. Arakelian and Y. R. Shen, Phys. Rev. Lett., 47, 1411 (1981).

6. I. C. Khoo and Y. R. Shen, Opt. Eng., 24, 579 (1985).

7. I. C. Khoo, Appl. Phys. Lett., 41, 909 (1982); I. C. Khoo, T. H. Liu, P. Y. Yan and J. Hou, Phys. Rev., A 29, 2756 (1984).

8. J. A. Martín-Pereda and F. J. López, Opt. Lett., 7, 590 (1982); J. A. MartínPereda, F. J. López, and M. A. Muriel, Mol. Cryst. Liq. Cryst., 99, 1 (1983).

9. F. J. López, Ph.D. Thesis, E. T. S. I. Telecomunicación, Madrid, (1983).

10. J. M. Otón, M. A. Muriel and J. A. Martín-Pereda, Presented at the XX Reunion Bienal Real Soc. Esp. Fis., 466 (1985).

11. J. M. Otón, R. Beltrán and J. A. Martín-Pereda, Mol. Cryst. Liq. Cryst., 127, $43(1985)$. 